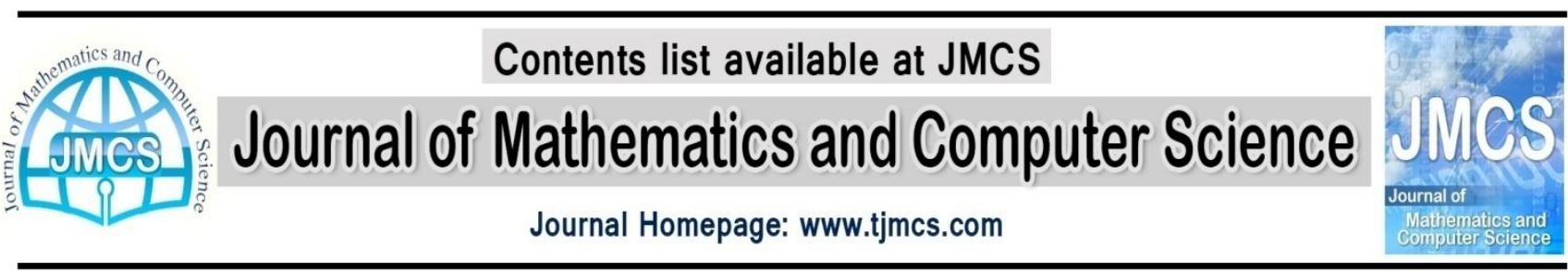

\title{
Direct and Fuzzy Transform Methods for the Stabilization Vibrations of a Damped Linear String
}

\author{
Rajib Ghosh $^{1, *}$, Ganesh .C. Gorain ${ }^{1,+}$, Samarjit Kar ${ }^{2}$ \\ ${ }^{1}$ Department of Mathematics, J. K. College, Purulia, West Bengal, 723101, India \\ rajibghosh0509@gmail.com \\ +goraing@gmail.com \\ ${ }^{2}$ Department of Mathematics, N. I. T, Durgapur, West Bengal, 723209, India \\ kar_s_k@yahoo.com
}

Article history:

Received January 2015

Accepted April 2015

Available online April 2015

\section{Abstract}

In this paper, the asymptotic behaviour of the vibrations of a damped linear string is studied. The exponential stability result of the overall system is obtain directly by means of an exponential energy decay estimate. A closed form approximate numerical result is constructed by fuzzy transform method to support and implement the stability result.

Keywords: Uniform stability, exponential energy decay estimate, Layapunov function, fuzzy transform method.

\section{MSC 2010: 35B35; 35L05; 35L10; 35L35; 35R13}

\section{Introduction and Mathematical Formulation}

Recently, studies on vibrational stability of different mechanical system have developed significantly due to increase of usage of flexible structures. The stabilization of the vibrations of a flexible structure is a problem of dynamical system governed by partial differential equations. Damping mechanism of passive type is a very common treatment of vibrational stability which uses a resistive device that absorbs vibration energy. In mathematical literature, the stabilities most commonly studied are asymptotic stability and uniform stability. A system is called asymptotic stable, if the solution of the system converges to zero as time tends to infinity. If this convergence is uniform with respect to all initial data, the system is called uniformly stable. Though the vibration of flexible structure are nonlinear in practice, linearized model are treated just for analytical approach, simplicity and for concise result. The theory of stability of a second order hyperbolic equation like wave equation has been treated earlier by several authors (cf. Lagnese [1], Komornik

[10], Shahruz [8], Nandi et al [13]). The most important fact for studying the stability of such system is to suppress the vibrations to assure a good performance of the overall system. The stability by means of the energy decay rate for the solution of internally damped wave equation in a bounded 
domain has been studied by Gorain [4]. Gorain [5] and Prasanta et al [14] have treated the case of Kirchhof type wave equation with Kelvin-Voigt model of viscoelasticity and then Gorain [3,6] and Siddartha et al [12] has extended the idea to the vibrations of inhomogeneous beams and strings. Recently, Rajib et al [7] have obtain the uniform stability result for the vibrations of a telegraph equation together with a numerical support by fuzzy transform method.

We observe keenly that knowledge on the study of dynamical system modeled by differential equations is sometimes incomplete or vague. For examples, knowledges of the parameters, initial conditions, boundary conditions, disturbances involved in the system are indistinct, in general. In fact, functional relationship connecting different parameter of a system does not charterized the whole set of system behaviour compatible with our partial knowledge. This idea leads all systems to Fuzzy Input Fuzzy Output (FIFO) systems. In this paper, we like to introduce and apply the idea of Stepnicka [17], Perfilieva et al [15] to a specific dynamical system that can be shown uniform stable without going through its analytical solutions. This techniques is based on two transforms: one direct fuzzy transform or, F-transform and another inverse F-transform. Practically, the technique based on this transform has gained importance due to its wide application on differential equations especially on partial differential equations.

The physical model considered here is the vibrations of a simple linear string having length $\mathrm{L}$ which is clamped at both ends. Suppose that it is made of a viscoelastic material of Kelvin-Voigt constitutive relation

(cf. Fung [2]). Consequently, the vibration of the string can be described by the following partial differential equation

$$
u_{t t}+\mu u_{t}=c^{2} u_{x x}, \text { in }(0, \mathrm{~L}) \times \mathbb{R}^{+}
$$

Where $\mathrm{c}$ is the wave velocity and $\mu$ be the coefficient of damping and $R^{+}=(0, \infty)$.

For a clamped string, the boundary conditions are obviously

$$
\mathrm{u}(0, \mathrm{t})=0, \mathrm{u}(\mathrm{L}, \mathrm{t})=0 \quad \text { on } \quad \mathbb{R}^{+}
$$

Let initially the string is set to vibrate with

$$
\mathrm{U}(\mathrm{x}, 0)=\mathrm{f}(\mathrm{x}), \quad u_{t}(\mathrm{x}, 0)=\mathrm{g}(\mathrm{x}) \quad \text { in }(0, \mathrm{~L}) .
$$

The function $\mathrm{f}(\mathrm{x})$ and $\mathrm{g}(\mathrm{x})$ are assume to be continuous upto second order partial derivatives over [0, $\mathrm{L}]$ so that the solution $\mathrm{u}(\mathrm{x}, \mathrm{t})$ is continuously differentiable over $(0, \mathrm{~L}) \times \mathbb{R}^{+}$.

Our aim of this work is to study the uniform stability result for the solutions of the mathematical problem (1) subject to the initial - boundary conditions (2) - (3). The uniform exponentially stability result is obtained by means of an explicit form of exponential energy decay estimate. For this, we adopt here a direct method by constructing suitable Layapunov functional related to the energy functional of the system. The detailed discussion of such method can be found in Komornik [9]. In the next step, we present an approximate closed form numerical scheme of the above problem using fuzzy transform of two variables following the idea of Stepnicka [18], Perfilieva et al [15]. Under this scheme the above system reduces to a set of discrete recursive equations which we are able to solve. 
The computational solutions are then plotted graphically for different values of the parameters and the initial conditions. Then verified the analytical result of uniform stability obtained directly is verified with these solutions.

To achieve the result, we now construct the energy of the system in the following.

\section{Energy of the System}

We define the energy $E(u(x, t))$ of the system (1)-(3) at any instant $t$ by

$$
E(u(t))=\frac{1}{2} \int_{0}^{L}\left[u_{t}^{2}+c^{2} u_{x}^{2}\right] d x, \quad \text { for all } t \geq 0 .
$$

Now, differentiating (4) with respect to t, we have

$$
\frac{d E}{d t}=\int_{0}^{L}\left[u_{t} u_{t t}+c^{2} u_{x} u_{x t}\right] d x
$$

Using (1) in (5) and applying the boundary conditions in (2), we get

$$
\frac{d E}{d t}=-\mu \int_{0}^{L} u_{t}^{2} d x \leq 0 \text { for all } t \geq
$$

0.

It follows from (6) that is the system (1)-(3) is energy dissipating and hence on integrating on it with respect to $t$ over $[0, t]$, the solution $\mathrm{u}(\mathrm{x}, \mathrm{t})$ satisfy energy estimate

$$
E(u(t))-E(u(0))=\int_{0}^{L} \int_{0}^{L} u_{\tau}^{2} d x d \tau \text { for all } t \geq 0,
$$

Where

$$
E(u(0))=\frac{1}{2} \int_{0}^{L}\left[g^{2}+c^{2} f_{x}^{2}\right] \mathrm{dx}
$$

In view of (7) and (8), we may conclude that if $f \in H_{0}^{L}(0, L)$ and $g \in L^{2}(0$, L), where

$$
H_{0}^{1}=\left\{\varphi: \varphi \in H^{1}(0, L), \varphi(0)=\varphi_{x}(0)=0\right\}
$$

is the subspace of classical Sobolev space $H^{1}(0, L)=\left\{\varphi: \varphi \in L^{2}(0, L), \varphi_{x} \in L^{2}(0, L)\right\}$ of real valued function of order one, then

$$
E(u(t)) \leq E(u(0))<\infty \text { for } t \geq 0 .
$$

\section{Uniform Stability Result by Direct Method}

Since the energy of the system (1)-(3) given by (4) is a dissipative function of time, so, naturally a question arises as to whether the energy decays with time exponentially or not and the affirmative answer is found in the following theorem. 
Theorem: If $\mathrm{u}(\mathrm{x}, \mathrm{t})$ be the solution of the system (1)-(3) with $(f, g) \in H_{0}^{1}(0, L) \times L^{2}(0, L)$ then the solution $\mathrm{u}(\mathrm{x}, \mathrm{t}) \rightarrow 0$ exponentially as $t \rightarrow \infty$, in other words, the energy functional given by (4) satisfies

$$
E(u(t)) \leq A e^{-\gamma t} E(u(0)), \quad t \geq 0
$$

For some reals $\gamma>0$ and $A>1$.

First, we need the following two inequalities.

For any real number $\alpha$, we have Young's inequality (cf. Mitrinovic et al [11])

$$
\int|f . g| d x \leq \frac{1}{2} \int\left[\alpha|f|^{2}+\frac{|g|^{2}}{\alpha}\right] d x .
$$

Poincare type Scheeffer's inequality (cf. Mitrinovic et al [11])

$$
\int_{0}^{L} u^{2} d x \leq \frac{L^{2}}{\pi^{2}} \int_{0}^{L} u_{x}^{2} d x
$$

As $\mathrm{u}(\mathrm{x}, \mathrm{t})$ satisfy the boundary conditions in (2).

To prove the above theorem, we proceed like Komornik [9], Gorain [3, 4]. Let us introduce an energy like Layapunov functional denoted by $\mathrm{V}(\mathrm{u}(\mathrm{t}))$ and is defined by

$$
V(u(t))=E(u(t))+\epsilon G(u(t)) \quad \text { for } \quad t \geq 0 \text {, }
$$

where $\varepsilon>0$ is a small real number and

$$
G(u(t))=\int_{0}^{L}\left[u u_{t}+\frac{\mu}{2} u^{2}\right] d x .
$$

Differentiating (14) with respect to $t$, we have

$$
\frac{d G}{d t}=\int_{0}^{L} u_{t}^{2} d x+\int_{0}^{L} u u_{t t} d x+\int_{0}^{L} \mu u u_{t} d x
$$

Using (1) and (2), we get

$$
\frac{d G}{d t}=\int_{0}^{L} u_{t}^{2} d x+c^{2} \int_{0}^{L} u u_{x x} d x
$$

Hence

$$
\frac{d G}{d t}=\int_{0}^{L} u_{t}^{2} d x-c^{2} \int_{0}^{L} u_{x}^{2} d x
$$

Using energy equation (4) in (17), we get 
(18)

$$
\frac{d G}{d t}=-2 E(u(t))+2 \int_{0}^{L} u_{t}^{2} d x
$$

Again by using the inequalities (11) and (12), we get

$$
\begin{aligned}
& \left|\int_{0}^{L} u u_{t} d x\right|=\frac{1}{c}\left|\int_{0}^{L}(c u) u_{t} d x\right| \\
& \leq \frac{1}{2 c}\left[\frac{\pi}{L} \int_{0}^{L} c^{2} u^{2} d x+\frac{L}{\pi} \int_{0}^{L} u_{t}^{2} d x\right] \\
& \leq \frac{1}{2 c}\left[\frac{\pi}{L} \frac{L^{2}}{\pi^{2}} \int_{0}^{L} c^{2} u_{x}^{2} d x+\frac{L}{\pi} \int_{0}^{L} u_{t}^{2} d x\right] \\
& =\frac{1}{2 c}\left[\frac{L}{\pi} \int_{0}^{L} c^{2} u_{x}^{2} \mathrm{dx}+\frac{L}{\pi} \int_{0}^{L} u_{t}^{2} d x\right] \\
& =\frac{1}{2 c} \frac{L}{\pi} \int_{0}^{L}\left[c^{2} u_{x}^{2}+u_{t}^{2}\right] d x \\
& =\frac{L}{\pi c} E(u(t)) \\
& =\lambda_{0} E(u(t)) \quad \text { for } t \geq 0 \text {, } \\
& \text { where }
\end{aligned}
$$

$$
\lambda_{0}=\frac{L}{\pi c}
$$

Also by using (12), we get

$$
\begin{aligned}
\int_{0}^{L} \frac{\mu}{2} u^{2} d x & =\frac{\mu}{2} \int_{0}^{L} u^{2} d x \\
& \leq \frac{\mu}{2} \frac{L^{2}}{\pi^{2}} \int_{0}^{L} u_{x}^{2} d x \\
& =\lambda_{1} E(u(t)) \text { for } t \geq 0,
\end{aligned}
$$

Where

$$
\lambda_{1}=\frac{\mu}{c^{2}} \frac{L^{2}}{\pi^{2}}
$$

Now, by (19) and (21), we estimate (14) as

$$
\rho_{0} E(u(t)) \leq G(u(t)) \leq\left(\lambda_{0}+\lambda_{1}\right) E(u(t)) \quad \text { for } t \geq 0 .
$$

Hence $\mathrm{V}(\mathrm{u}(\mathrm{t}))$ as defined in (13) can be estimated as

$$
\left(1-\lambda_{0} \epsilon\right) E(u(t)) \leq G(u(t)) \leq\left[1+\left(\lambda_{0}+\lambda_{1}\right) \epsilon\right] E(u(t)) \quad \text { for } \quad t \geq 0 .
$$

We choose $\left(1-\lambda_{0} \epsilon\right)>0$ that is $\epsilon<\frac{1}{\lambda_{0}}$ so that $V(u(t)) \geq 0$ for $t \geq 0$. 
Now, taking the time derivative of (13) and applying the results (6) and (18), we get

$$
\begin{aligned}
\frac{d V}{d t}=\frac{d E}{d t} & +\epsilon \frac{d G}{d t} \\
& =-\mu \int_{0}^{L} u_{t}^{2} d x-2 \epsilon E(u(t))+2 \epsilon \int_{0}^{L} u_{t}^{2} d x \\
& =-2 \epsilon E(u(t))-(\mu-2 \epsilon) \int_{0}^{L} u_{t}^{2} d x \\
& =-\frac{2 \epsilon}{1+\left(\lambda_{0}+\lambda_{1}\right) \epsilon} V(u(t))-(\mu-2 \epsilon) \int_{0}^{L} u_{t}^{2} d x
\end{aligned}
$$

Since $\epsilon$ is small, we assume that

$$
\epsilon<\epsilon_{0}=\min \left[\frac{1}{\lambda_{0}}, \frac{\mu}{2}\right]
$$

Hence from (24), we get the differential inequality

$$
\frac{d V}{d t}+\gamma V(u(t)) \leq 0 \text { for } t \geq 0
$$

Where

$$
\gamma=\frac{2 \epsilon}{1+\left(\lambda_{0}+\lambda_{1}\right) \epsilon}
$$

Multiplying (26) by $e^{\gamma t}$ and integrating over $[0, \mathrm{t}]$ for any $t \in \mathbb{R}^{+}$, we get

$$
V(u(t)) \leq e^{-\gamma t} V(u(0))
$$

Applying the inequality (23) in (28), we get

Thus

$$
E(u(t)) \leq \frac{1+\left(\lambda_{0}+\lambda_{1}\right) \epsilon}{1-\lambda_{0} \epsilon} e^{-\gamma t} E(u(0))
$$

Where

$$
E(u(t)) \leq A e^{-\gamma t} E(u(0)), \quad t>0
$$

Thus the result is obtained directly and the theorem is proved. The above result shows the uniform exponential stability of the system (1)-(3) by means of an exponential energy decay estimate. Hence the solution of the system (1)-(3) decays exponentially with time, that means, $u(x, t) \rightarrow 0$ as $t \rightarrow+\infty$ for every $\left(u_{0}, u_{1}\right) \in H_{0}^{1}(0, L) \times L^{2}(0, L)$

We shall now verify the above result by a closed form numerical scheme using fuzzy transform technique. To study the scheme, we first introduce basic ideas of fuzzy transform or, F-transform. 


\section{F-Transforms for Functions}

In this section we proceed like Perfilieva [15] and Stepnicka [18]. This technique is rather numerical than linguistic for computing solutions on the basis of fuzzy approximation. An interval $[a, b]$ of real numbers has been used as common domain of all functions.

Let $x_{i}=a+b(i-1), i=1,2, \ldots, n$ be nodes on [a, b], where $h=\frac{b-a}{n-1}, n>2$. We say that functions $A_{1}(x), A_{2}(x), \ldots, A_{n}(x)$ defined on $[\mathrm{a}, \mathrm{b}]$ are basis functions, if each of them fulfils the following conditions:
i) $\quad A_{i}:[a, b] \rightarrow[0,1], A_{i}\left(x_{i}\right)=1$,
ii) $\quad A_{i}(x)=0$ if $x \notin\left(x_{i-1}, x_{i}\right)$, when $x_{0}=a, x_{n+1}=b$,
iii) $\quad A_{i}(x)$ is continuous,
iv) $\quad A_{i}(x)$ strictly increases on $\left[x_{i-1}, x_{i}\right]$ and strictly decreases on $\left[x_{i}, x_{i+1}\right]$
v) $\quad \sum_{i=1}^{n} A_{i}(x)=1$ for all $x \in[a, b]$,
vi) $\quad A_{i}\left(x_{i}-x\right)=A_{i}\left(x_{i}+x\right)$, for all $x \in[0, h], i=1,2, \ldots, n-1, n>2$,
vii) $\quad A_{i+1}(x)=A_{i}(x-h)$, for all $x \in[a+h, b], i=2, \ldots \ldots, n-2, n>2$,

Zadeh [19] introduce the concept of fuzzy sets via membership function as mathematical means of describing vagueness in linguistic. In last two decay many theoretical as well as numerical developments in fuzzy logic took place among the researchers of mathematical communities. In this case, we say that basic functions $A_{1}(x), \ldots, A_{n}(x)$ determine a uniform fuzzy partition of interval [a, b]. In other words, basic functions are the fuzzy sets determining a uniform fuzzy partition of real interval [a, b]. Moreover, each basic function $A_{i}(x)$ can be viewed as a fuzzy set "approximately $x_{i}$ ". The technique of fuzzy transforms is based on two transforms: the direct one and the inverse one. At first, we transform an element of space of continuous functions $C[a, b]$ to a vector which serves us as its discrete representation.

Let $f(x)$ be a continuous function on [a, b] determining a uniform fuzzy partition of $[\mathrm{a}, \mathrm{b}]$. If we set

$$
F_{i}=\frac{\int_{a}^{b} f(x) A_{i}(x) d x}{\int_{a}^{b} A_{i}(x) d x}, i=1,2, \ldots, n .
$$

Then the n-tuple of real number $\left[F_{1}, F_{2}, \ldots, F_{n}\right]$ called the direct F-transform of ' $\mathrm{f}$ ' with respect to the basic functions $A_{1}(x), \ldots, A_{n}(x)$. Each $F_{i}$ is called component of f-transform and the totality can be viewed as an aggregate representation of the function $\mathrm{f}$. Moreover, they will be used in a construction of a simplified continuous approximate representation of $\mathrm{f}$. On the other hand, if $F_{n}[f]=$ $\left[F_{1}, F_{2}, \ldots F_{n}\right]$ be the F-transform of $\mathrm{f}$ with respect to the basic function $A_{1}, A_{2}, \ldots, A_{n}$. Then the function

$$
f_{n}^{F}(x)=\sum_{i=1}^{n} A_{i}(x) F_{i}
$$

is called the inverse F-transform. 


\section{Closed Form Numerical Scheme}

In this section, our purpose is to solve system of equation (1)-(3) that means, the following Initial Boundary value problem:

$$
\begin{gathered}
u_{t t}+\mu u_{t}=c^{2} u_{x x}, \quad(0, L) \times(0, \infty) \\
u(x, 0)=f(x), u_{t}(x, 0)=g(x), \quad x \in(0, L) \\
u(0, t)=0, u(L, t)=o, \quad t \in(0, \infty) .
\end{gathered}
$$

Using F-transform method.

By applying F-transform in equation (32) is transformed into the following algebraic equation

$$
F^{2}\left[u_{t t}\right]=c^{2} F^{2}\left[u_{x x}\right]-\mu F^{2}\left[u_{t}\right]
$$

Where $F^{2}\left[u_{t t}\right], F^{2}\left[u_{x x}\right], F^{2}\left[u_{t}\right]$ are the matrices of the F-transform components of $u_{t t}, u_{x x}, u_{t}$ given by

$$
\begin{aligned}
& F^{2}\left[u_{t t}\right]=\left[\begin{array}{cccc}
u_{t t}^{11} & u_{t t}^{12} & \cdots & u_{t t}^{1 m} \\
\vdots & & \vdots \\
u_{t t}^{n 1} & u_{t t}^{n 2} & \cdots & u_{t t}^{n m}
\end{array}\right], \\
& \text { and } \quad F^{2}\left[u_{x x}\right]=\left[\begin{array}{cccc}
u_{x x}^{11} & u_{x x}^{12} & \cdots & u_{x x}^{1 m} \\
\vdots & & \vdots \\
u_{x x}^{n 1} & u_{x x}^{n 2} & \cdots & u_{x x}^{n m}
\end{array}\right], \\
& F^{2}\left[u_{t}\right]=\left[\begin{array}{cccc}
u_{t}^{11} & u_{t}^{12} & \cdots & u_{t}^{1 m} \\
\vdots & & \vdots \\
u_{t}^{n 1} & u_{t}^{n 2} & \cdots & u_{t}^{n m}
\end{array}\right]
\end{aligned}
$$

To determine the matrices $F^{2}\left[u_{t t}\right], F^{2}\left[u_{x x}\right]$ and $F^{2}\left[u_{t}\right]$, we replace the partial derivatives in (32) by approximation as

$$
\begin{gathered}
u_{t t} \approx \frac{u(x, t+k)-2 u(x, t)+u(x, t-k)}{k^{2}}, \\
u_{x x} \approx \frac{u(x+h, t)-2 u(x, t)+u(x-h, t)}{h^{2}}, \\
u_{t} \approx \frac{u(x, t+k)-u(x, t)}{k} .
\end{gathered}
$$

Next, we can approximate $u_{t t}^{i j}$ as follows:

$$
u_{t t}^{i j}=\frac{\iint \frac{\partial^{2} u}{\partial t^{2}}(x, t) A^{i}(x) B^{j}(t) d x d t}{\iint A^{i}(x) B^{j}(t) d x d t}
$$




$$
\begin{aligned}
& \approx \frac{\iint\left[\frac{u(x, t+k)-2 u(x, t)+u(x, t-k)}{k^{2}}\right] A^{i}(x) B^{j}(t) d x d t}{\iint A^{i}(x) B^{j}(t) d x d t} \\
& =\frac{1}{k^{2}}\left[u^{i, j+1}-2 u^{i, j}+u^{i, j-1}\right] .
\end{aligned}
$$

Similarly,

$$
u_{x x}^{i j}=\frac{1}{h^{2}}\left[u^{i+1, j}-u^{i, j}+u^{i-1, j}\right]
$$

$$
u_{t}^{i j}=\frac{1}{k}\left[u^{i, j+1}-u^{i, j}\right]
$$

By the use of (34), (35) and (36), we come to the following recursive equation

$$
\frac{1}{k^{2}}\left[u^{i, j+1}-2 u^{i, j}+u^{i, j-1}\right]=\frac{c^{2}}{h^{2}}\left[u^{i+1, j}-u^{i, j}+u^{i-1, j}\right]-\frac{\mu}{k}\left[u^{i, j+1}-u^{i, j}\right]
$$

Or, $\left(1+r_{2}\right) u^{i, j+1}=r_{1}^{2} u^{i+1, j}+\left(2-2 r_{1}^{2}+r_{2}\right) u^{i, j}+r_{1}^{2} u^{i-1, j}-u^{i, j-1}$,

where $r_{1}=\frac{c k}{h} \quad$ and $r_{2}=\mu k$.

Now the above can be written as

$$
\left.u^{i, j+1}=A\left[u^{i+1, j}+u^{i-1, j}\right]+B u^{i, j}-C u^{i, j-1}\right],
$$

$$
i=1,2, \ldots \ldots \ldots \ldots, m-1, \quad j=0,1,2, \ldots \ldots \ldots, n-1,
$$

where $\quad A=\frac{r_{1}^{2}}{1+r_{2}}, \quad B=\frac{2-2 r_{1}^{2}+r_{2}}{1+r_{2}}, \quad C=\frac{1}{1+r_{2}}$.

In particular $j=0$, then the above equation becomes

$$
u^{i, 1}=A\left[u^{i+1,0}+u^{i-1,0}\right]+B u^{i, 0}-C u^{i,-1} .
$$

The unknown $u^{i,-1}$ for $i=0,1,2, \ldots, m$ occurring in recursive equation (38) can be obtained from the second initial condition $u_{t}(x, 0)=g(x)$ in (32) by the following difference scheme.

$$
\frac{\partial u}{\partial t} \approx \frac{u(x, t+k)-u(x, t)}{k} .
$$

The above scheme for $\mathrm{k}=1$, leads to

$$
\begin{gathered}
\frac{u^{i, 1}-u^{i,-1}}{2 k}=g(i h) \\
\text { or, } u^{i,-1}=u^{i, 1}-2 k g(i h) .
\end{gathered}
$$

Putting the values of $u^{i,-1}$ in equation (38), we have for $i=1,2, \ldots, m-1$, 


$$
u^{i, 1}=\frac{A}{1+C}\left[u^{i+1,0}+u^{i-1,0}\right]+\frac{B}{1+C} u^{i, 0}+2 \frac{C}{1+C} k g(i h)
$$

Thus, we can obtain all the values of $u^{i j}$ for $\mathrm{j}=1$ level, since $u^{i, 0}=f(i h)$ are known for $i=$ $0,1, \ldots \ldots \ldots, m$, from the given function $f(x)$.

For second and higher order levels, we put $j=1,2, \ldots, n-1$ in the recursive relation (37), where

$$
u(0, j)=0, \quad u(m, j)=0, \quad j=0,1, \ldots, n-1
$$

Followed from the boundary conditions in (32).

Applying the above computational scheme with different values of the parameters, the dynamical responses of the solution are shown in the following figures.

The figure 5.1 is obtained with parameters $c=1, \mu=0.2, k=0.05, h=0.1, f(x)=\operatorname{Sin}(\pi x)$, $g(x)=0.015 x$ over the interval $[0,4]$.

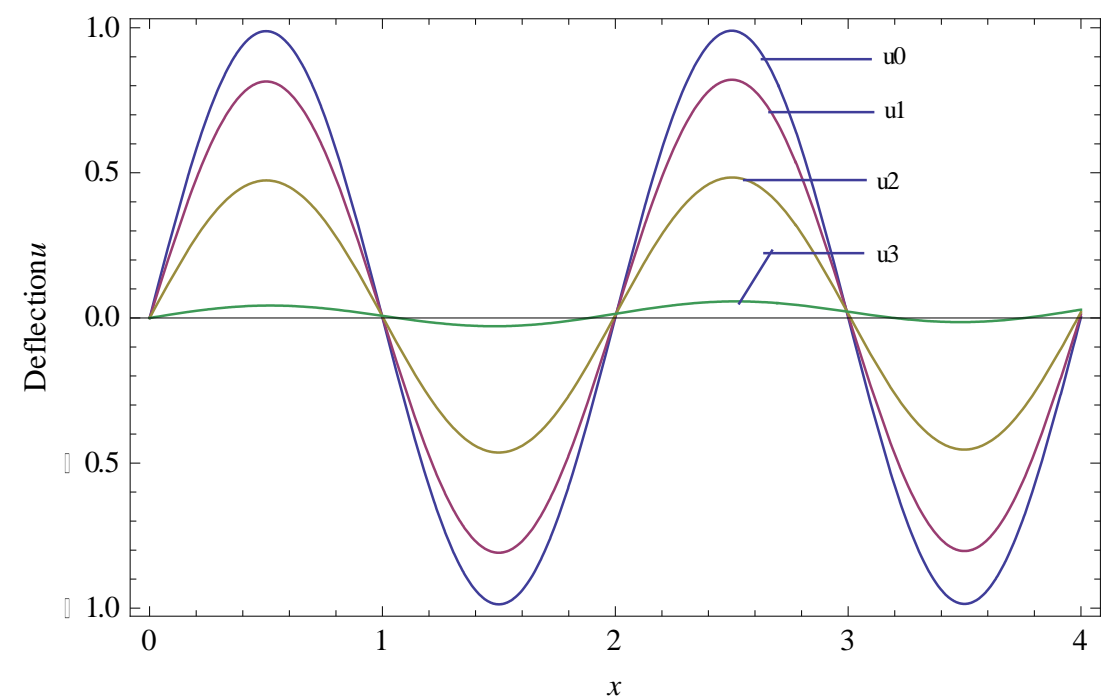

Fig 5.1: Approximate deflections $\mathrm{u}(\mathrm{x}, \mathrm{t})$ of the string for different values of time. $u_{0}, u_{1}, u_{2}, u_{3}$ are the deflections of the string for the numerical value of $t=0, t=0.20, t=0.30, t=0.5$ respectively.

The figure 5.2 is obtained with parameters $c=1.2, \mu=0.15, k=0.04, h=0.1, f(x)=\frac{x \operatorname{Cons}(\pi x)}{5}$, $g(x)=0.01 x$ over the interval $[0,4.5]$. 


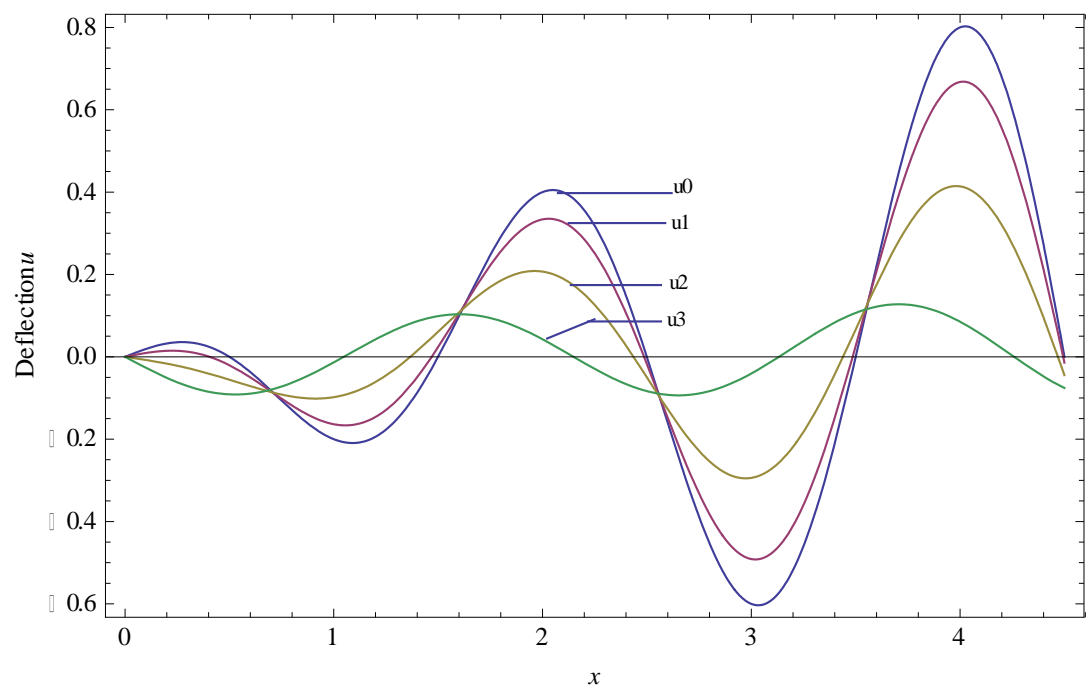

Fig 5.2: Approximate deflections $\mathrm{u}(\mathrm{x}, \mathrm{t})$ of the string for different values of time. $u_{0}, u_{1}, u_{2}, u_{3}$ are the deflections of the string for the numerical value of $t=0, t=0.16, t=0.28, t=0.4$ respectively.

The figure 5.3 is obtained with parameters $c=2, \mu=0.25, k=0.02, h=0.15, f(x)=$ $0.5 e^{0.1 x} \sin (\pi x), g(x)=0.001 x$ over the interval $[0,4]$.

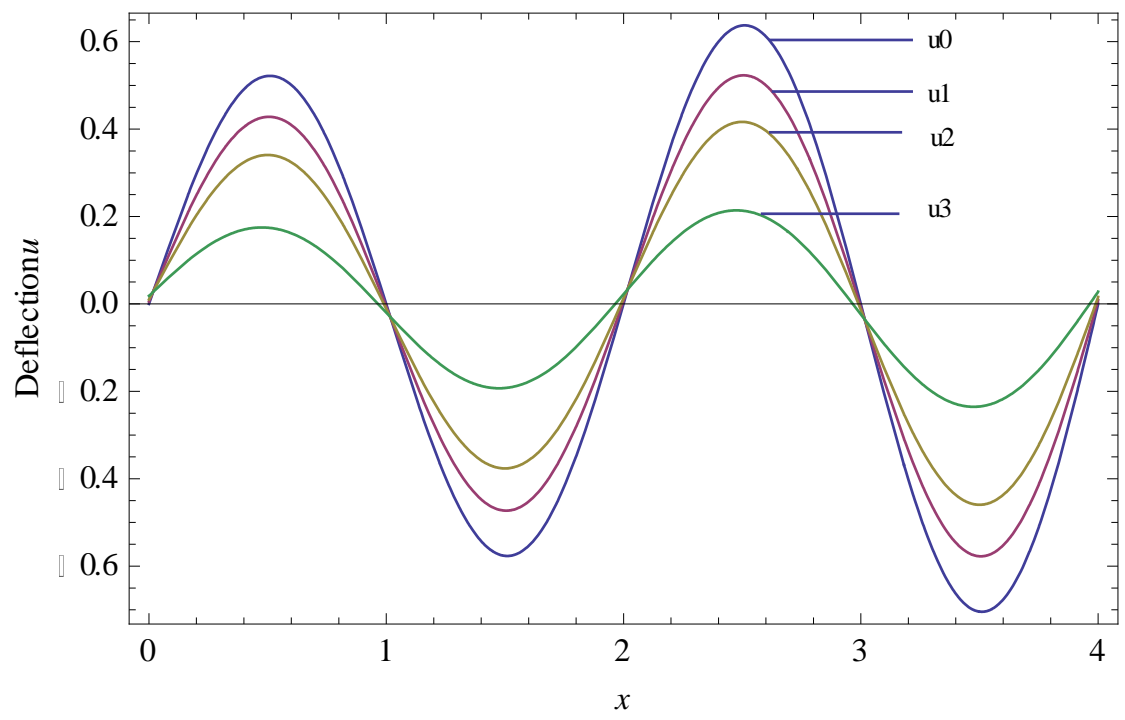

Fig 5.3 : Approximate deflections $\mathrm{u}(\mathrm{x}, \mathrm{t})$ of the string for different values of time. $u_{0}, u_{1}, u_{2}, u_{3}$ are the deflections of the string for the numerical value of $t=0, t=0.08, t=0.14, t=0.20$ respectively.

We observe that the above numerical results through different graphs, the computational results obtained by F-transform method satisfy the analytical result of uniform exponential decay of solution that was obtained directly. Thus, the computational result corresponds almost exactly to the theoretical stability result so obtained.

\section{Conclusion}

This study deals a comparison between the mathematical stability result obtained directly by means of an uniform exponential decay estimate and the numerical stability result obtained by F-transform method for the vibration of a string modelled by the partial differential equation (1). The numerical 
solutions of the system shown in the figures exhibits a coincidence to the decay of solution of the system (1)-(3) with time. This highly signifies our study in this work.

\section{References}

[1] G. Chen, "A note on the boundary stabilization of the wave equation", SIAM J. Control Optim. 19, (1981) pp. 106-113.

[2] Y. C. Fung, "Foundations of solid mechanics", Prentice- Hall, New Delhi (1968).

[3] G. C. Gorain, "Stabilization of a Quasi-linear vibrations of an Inhomogeneous Beam", IEEE Trans.Automat.Contr.Vol.52 (2007), pp-1690-1695.

[4] G. C. Gorain, "Exponential Energy Decay Estimate for the solutions of Internally Damped Wave equation in a Bounded domain", J.Math.Anai.Appl, 216 (1997), pp.510-520.

[5] G. C. Gorain, "Exponentially Energy Decay Estimate for the Solutions of n-Dimensional Kirchhoff type Wave Equation", Applied Mathematics and Computative, 177 (2006), pp.235 242.doi:10.1016/j. amc. 2005.11.03.

[6] G. C. Gorain, "Exponential stabilization of longitudinal vibration of an inhomogeneous beam" (2014).

[7] R. Ghosh, S. Chowdhury, G. C. Gorain, S. Kar, "Uniform stabilization of the telegraph equation with a support by fuzzy transform method", QScience connect 2014:19(2014).

[8] V. Komornik, "Rapid Boundary Stabilization of Wave Equations", SIAM Journal of control and Optimization, 29 (1991), pp.197-208.doi:10.1137/0329011.

[9] V. Komornik, "Exact Controllability and Stabilization", the Multiplier Method, John WileyMasson (1994), Paris.

[10] J. Lagnese, "Note on boundary stabilization of wave equation", SIAM J. Control Optim. 26 (1988), pp. 1250-1256.

[11] D. S. Mitrinovic, J. E. Pecaric, A. M. Fink, "Inequalities involving functions and their integrals and derivatives". Dordrecht, The netherlands: Kluwer (1991).

[12] S. Mishra, G. C. Gorain, "Stability of an inhomogeneous damped vibrating string", Application and applied mathematics, Vol 9(1) (2014), pp. 435-448.

[13] P. K. Nandi, G. C. Gorain, S. Kar, "Boundary stabilization of torsional vibration of a solar panel", Appl. Math, 7 (2012), pp. 455-463.

[14] P. K. Nandi, G. C. Gorain, S. Kar, "Stability of vibrations for some Kirchoff equations with dissipating", Application of Mathematics, vol-59(2) (2014), pp. 205-215.

[15] I. Perfilieva, E. Chaldeeva, "Fuzzy Transformation and its Applications", In Proc.Of the 4th czech-Japan Seminar on Data Analysis and Decision Making under Uncertainty, 124 (2001). pp.116-S.

[16] M. Saharuz, "Bounded-Input-Bounded-Output Stability of Damped Non-Linear String”, IEEE.Trans.Auto.Control, 41 (1996), pp. 1179-1182.

[17] M. Stepnicka, R. Valasek, "Fuzzy Transforms For Functions With Two Variables", In:Proc.Of the 6th Czech-Japan Seminar On Data Analysis and Decision making Under Uncertainty, Valtice, Czech Republic, Sept.20-23 (2003) pp. 100-107.

[18] M. Stepnicka, R. Valasek, "Fuzzy Transforms and Their Application to Wave Equation", Journal of Electrical Engineering, 55(12/s), (2004), pp.7-10.

[19] L. A. Zadeh, "Fuzzy sets and systems, System Theory (Fox J., ed.), Microwave Research Institute Symposia Series XV', Polytechnic Press, Brooklyn, NY, 29-37. Reprinted in Int. J. of General Systems, 17, 1990 (1965), 129-138. 